\title{
Current trends in midface reconstruction and use of virtual surgical planning
}

\author{
Larissa Sweeny ${ }^{1}$, Andrew R. Fuson', Joseph M. Curry ${ }^{2}$ \\ 'Department of Otolaryngology - Head and Neck Surgery, Louisiana State University Health Science Center - New Orleans, New \\ Orleans, LA 70112, USA. \\ ${ }^{2}$ Department of Otolaryngology - Head and Neck Surgery, Thomas Jefferson University, Philadelphia, PA 19107, USA.
}

Correspondence to: Dr. Larissa Sweeny, Department of Otolaryngology - Head and Neck Surgery, Louisiana State University Health Science Center - New Orleans, 533 Bolivar Street, Ste 566, New Orleans, LA 70112, USA. Email: Iswee1@lsuhsc.edu

How to cite this article: Sweeny L, Fuson AR, Curry JM. Current trends in midface reconstruction and use of virtual surgical planning. Plast Aesthet Res 2021;8:18. https://dx.doi.org/10.20517/2347-9264.2020.228

Received: 29 Dec 2020 First Decision: 7 Feb 2021 Revised: 8 Feb 2021 Accepted: 22 Feb 2021 Available online: 18 Mar 2021

Academic Editor: Matthew Spector Copy Editor: Xi-Jun Chen Production Editor: Xi-Jun Chen

\begin{abstract}
Virtual surgical planning (VSP) applicability has recently expanded to include midface reconstruction with free tissue transfer. For the midface, an area that is crucial both functionally and aesthetically, even minor reconstructions (in the millimeter range) can make profound differences in form and function. The use of VSP allows the surgeon to improve accuracy and precision in areas where millimeters drastically impact outcomes. This review focuses on complex midface reconstruction requiring free tissue transfer and assesses the role of VSP in this patient population.
\end{abstract}

Keywords: Head and neck reconstruction, free flap, midface, virtual surgical planning

\section{INTRODUCTION}

Virtual surgical planning (VSP) utilizes computer-aided design and manufacturing. VSP encompasses the utilization of computer software for operative planning and the creation of patient-specific models, guides, and hardware. It is considered by many authors to be advantageous for preoperative assessment and surgical planning, resulting in shorter operative time and improved outcomes ${ }^{[1-10]}$. While its most utilized indication in the head and neck region has historically been for mandibular reconstruction, VSP has gained recent interest for use in midface reconstruction. 
Reconstruction of the midface is considered complex due to its intricate three-dimensional (3D) structure and its importance in speech, expression, aesthetics, and mastication. The midface contributes to facial contour, houses the maxillary dentition, supports the orbit and anterior skull base, and anchors muscles of mastication and facial expression. As a result, common complications seen following midface reconstruction include diplopia, ectropion, enophthalmos, velopharyngeal insufficiency, hardware exposure, hardware extrusion, and fistula formation.

One advantage of utilizing VSP for preoperative planning is the ability to visualize the surgical defect and rotate the facial skeleton in three dimensions. This allows the surgeon to better conceptualize the defect as well as determine the best orientation of the donor tissue to maximize cosmetic and functional outcomes. VSP provides a better understanding of the underlying shape of the recipient and donor bones, and aids in planning osteotomies that maximize bone-to-bone contact between the donor and recipient site. Improved bone apposition correlates with higher rates of bone union, and subsequently, lower rates of complications and improved cosmetic outcomes. This review will focus on complex midface reconstruction requiring free tissue transfer and assess the evolving role of VSP in this patient population.

\section{RECIPIENT AND DONOR SITE CONSIDERATIONS}

The complexity of the midface structure and its importance to a multitude of functions present the reconstructive surgeon with unique challenges. The greater the ability of the surgeon to recreate what is lost, the better the clinical outcomes for patients. The use of VSP allows the surgeon to improve accuracy and precision in areas where millimeters drastically impact outcomes.

The midface is composed of the palate, alveolus, paranasal sinuses, nasal cavity, and orbital cavity. The bones of the midface include the maxilla, ethmoid, nasal, palatine, and zygoma. Depending on the defect, goals of midface reconstruction may include closure of fistulas, restoration of soft tissue volumetric loss and symmetry, prevention of velopharyngeal insufficiency, orbital positioning, dental rehabilitation, and restoration of a bony framework for function and soft tissue support ${ }^{[2]}$. Cosmetically, projection is an important element to be factored into the reconstructive plan.

Restoration of the lost facial buttresses is paramount to success as they provide the framework and stability for the reconstructed midface. The horizontal buttresses make up the roof and floor of the midface, while the vertical buttresses make up the anterior, posterior, lateral, and medial walls. The anterior alveolus provides a framework for the maxillary dentition and the premaxilla provides support for the nose and has important aesthetic implications.

When selecting a donor site, the reconstructive surgeon needs to assess the defect and determine the goals of reconstruction. The bony reconstruction provides support to limit contracture and improve facial contouring. Therefore, determining which facial support mechanisms and structures have been affected is imperative. In addition to the bony framework, the volume of soft tissue lost must be taken into consideration by the reconstructive surgeon.

The free tissue donor sites available for reconstruction vary in morphological outcome $e^{[11]}$. Reconstruction of simple ostial midface defects may be achieved with the osteocutaneous radial forearm free flap. More complex reconstructions of the midface often include the restoration of numerous buttresses; the most common donor sites selected in these instances are the fibula or scapula. If the alveolus is involved, dental rehabilitation should be considered when selecting a donor site. Dental implantation success rates are similar between fibular and scapular free flaps ${ }^{[12]}$. 
The infraorbital curvature can be difficult to reproduce with any donor site. Custom orbital floor implants can be used with the addition of a soft tissue free flap to provide coverage and protection of the implant from exposure to the nasal flora. Potential donor sites for soft tissue coverage include the anterior lateral thigh or radial forearm, depending on volumetric needs. Adequate soft tissue coverage is crucial to prevent exposure of the implant to bacteria and chronic infection, leading to overlying skin breakdown, fistula formation, and extrusion of hardware.

\section{Radial forearm}

The radial forearm free flap (RFFF) is a fasciocutaneous flap that does not contain muscle. As such, it has better volumetric stability. It is pliable and ideal for palate reconstruction as it has limited interference with the retained mobility of the residual soft palate. In addition, it has a long pedicle that allows for tension-free anastomosis in the neck. The pedicle may also be harvested with additional adipose as needed for further volumetric contouring. Reconstruction of simple ostial midface defects may be achieved with the osteocutaneous radial forearm free flap (OCRFFF), and its ideal use includes defects limited to the alveolar ridge, infrastructure maxilla, or orbital $\operatorname{rim}^{[13]}$. Limitations of the OCRFFF include limited bone length (10$12 \mathrm{~cm}$ ), little soft tissue volume, and lack of bone stock for dental implant integration.

\section{Anterolateral thigh}

The anterolateral thigh (ALT) free flap can be used when large volumes of soft tissue loss are anticipated. The fat content of the flap allows for more predictable long-term volumetric retention as compared to myofascial or myocutaneous alternatives. However, it does not contain a bony component, therefore limiting its utility in midface buttress reconstruction and projection. It can be used to provide coverage of custom-made orbital rim implants in the setting of an orbital rim reconstruction. An additional scenario where the ALT free flap may be utilized includes large resections involving orbital exenteration in which a large amount of soft tissue may be required to obliterate the sinus cavities.

\section{Fibula}

The fibula is a versatile flap for midface reconstruction. Since it can be harvested as a long vascularized bone, the fibula may be used in scenarios when multiple osteotomies are required to restore the facial skeleton and contouring. Like the fasciocutaneous RFFF, the skin paddle of the fibula does not contain muscle. Consequently, this allows for greater volumetric stability over time. The soft tissue component can, however, be limited and may be inadequate for large volumetric soft tissue loss. The fibula bone harvested is bicortical and therefore allows for dental implant integration.

In order to utilize the fibula for reconstruction and midface defects, multiple osteotomies and acute angles are often required to achieve ideal contouring and support. The utilization of computer-assisted design or VSP has allowed surgeons to achieve this with greater accuracy ${ }^{[5,7,9,14]}$. It is generally recommended that an osteotomy should retain at least 2-3 centimeters of the bone to maintain the periosteal blood supply to the bone segment. However, few publications have noted that they consistently used 1.5 centimeters bone segments with flap success ${ }^{[7,15]}$.

It is important to consider the amount of bone stock required to restore the facial skeleton and buttress. As the number of osteotomies and bone length increase, the pedicle becomes shorter. A three-buttress defect is less ideal for a fibula free flap given the number of osteotomies required and the subsequent shortening of the pedicle length. In addition, with each additional osteotomy, there is an increased risk of osseointegration failure and subsequent malunion or nonunion ${ }^{[16]}$. Over time, this can manifest as hardware instability, extrusion, or fistula formation. 


\section{Subscapular}

The subscapular system of chimeric free flaps is extremely versatile. It can provide ample soft tissue, muscle, and bone. The scapula bone can be elevated with the thoracodorsal pedicle (tip), the circumflex scapular pedicle (lateral border), or both. If additional bone is required, the serratus muscle and rib can be harvested in conjunction with the flap. The latissimus dorsi can be utilized in conjunction with the scapula for added versatility. Multiple combinations of chimeric components, adequate bone for the majority of midface defects, and the predictable anatomy has resulted in the subscapular system being utilized with increasing frequency for midface reconstruction ${ }^{[17]}$.

The shape and natural contour of the scapula bone make it ideal for midface reconstruction, in particular, the anterior maxillary face and alveolar ridge. The scapula can be used to recreate the orbital rim and nasal aperture as well. The scapula tip has excellent morphologic consistency with the palate or orbital floor (horizontally oriented), or the anterior maxillary face (vertically oriented ${ }^{[18]}$; it can therefore be used for reconstructions of the hard palate or the surface of the anterior maxillary wall. When orienting the scapular tip in a horizontal plane, it is recommended that a plate be secured anterior to the remaining maxilla, posterolateral to the remaining zygoma, and posteriorly to the remaining contralateral hard palate to prevent inferior displacement of the scapula tip over time. When orienting the scapular tip in the vertical position, the maxillary sinus requires obliteration with a soft tissue to prevent an oral-nasal fistula. For defects involving the orbital rim and/or zygoma, the lateral border of the scapula and the scapula tip can be harvested as a single segment to reconstruct the lateral orbital rim, the zygoma (lateral border), and the anterior maxillary face (tip).

Adding to the versatility of the subscapular systems is the variety of soft tissue paddles that can be incorporated. These include abundant soft tissue in the form of fasciocutaneous, myocutaneous, or myofascial paddles. These soft tissue paddles can be used for complex volumetric reconstruction and allow for the most ideal perforator orientation.

Dental implantation into the scapula free flap has been reported to have success rates as high as those found with dental implantation into the fibula free flap ${ }^{[19,20]}$. Historically, dental implants into the scapula have been performed secondarily. Recently there are reports of immediate dental implantation into a scapula free flap $^{[18]}$.

A disadvantage of the lateral border and subscapular circumflex system is the short pedicle length, which may require vein grafts to achieve a tension-free anastomosis. The scapula tip and latissimus dorsi muscle originate from the thoracodorsal system, and as such, can be harvested with a longer pedicle length. It has been reported that the thoracodorsal system can be utilized to harvest up to $10 \mathrm{~cm}$ of lateral border of the scapula, increasing the available pedicle length ${ }^{[21]}$. Using this technique, the angular artery is utilized to supply the lateral border of the scapula as well as the scapular tip. While anatomic studies have demonstrated only a short segment of the lateral border receiving its principal vascularity from the angular artery, up to $74 \%$ of patients demonstrated evidence of bony union on imaging at one year postoperatively using this technique ${ }^{[21]}$. Additional critiques of the scapular flap include longer harvest times and patient positioning intraoperatively, which can either be a prone (requiring repositioning and longer operative duration) or a partial or tilted lateral decubitus position (allows for simultaneous harvest ${ }^{[22]}$.

\section{TECHNICAL CONSIDERATIONS}

To best utilize computer-assisted design, a fine-cut CT scan (one millimeter or less thickness) of the face and donor site is optimal. The software then utilizes data from the patient imaging to create a $3 \mathrm{D}$ rendering. 
If outsourcing, the surgeon will conference with the engineer to plan the procedure. This allows for the generation of patient-specific cutting guides and reconstructive plates. With the aid of cutting guides, osteotomies with maximum points of bony contact can be planned. These patient-specific plates, particularly for the midface, may be designed to have a stronger grade than plates that are traditionally bent intraoperatively. Another advantage of custom plates is that engineers may assess the thickness of the donor and recipient's bone to allow the planning of screw placements in areas with adequate bone thickness. This improves the stability of the screws and reduces the likelihood of screw or plate extrusion in the future. VSP and customizable plates also allow for the placement of hardware away from suture lines. This limits the potential exposure of the hardware to bacteria which may weaken the incision line over time resulting in potential fistula formation, hardware exposure, or hardware extrusion. While good results can be achieved through freehand creation of osteotomies, several publications have found that there is improved bone to bone contact accuracy when VSP is utilized ${ }^{[1,18,23]}$.

During the design session, visualization in the three planes, and alignment to the native midface and donor bone should be taken into consideration to ensure adequate contouring. Standard cutting guides used for bone reconstruction are shaped to the bone to be harvested and secured directly to the donor bone intraoperatively. This guides the cutting saw to divide the bone at appropriate angles. The scapula bone has several muscular attachments along its border which are crucial for bone viability and make it less amenable to cutting guides. To accommodate these muscle attachments, the guides can be designed such that they are elevated at a distance away from the bone. In addition, a cutting guide can be designed to be used with overlay technique. The overlay design can accommodate various thicknesses of muscle and allows the guide to be used as a template that is held in place. The saw can be guided to follow along its surface to ensure proper orientation and alignment.

Midface reconstructive examples can be seen in Figures 1-3. Figures 1 and 2 represent midface reconstructions utilizing VSP. For comparison of accuracy and fit, a midface reconstruction that was performed without VSP, due to a rapidly enlarging malignancy, is shown in Figure 3. Figures 1 and 2 demonstrate the use of the thin portion of scapula bone to create an orbital floor sling. The bone segment is secured with mini plates that are bent at a 90-degree angle and attached to the portion of the scapula bone to recreate the anterior maxillary face.

\section{SECONDARY RECONSTRUCTION}

VSP can be particularly advantageous in secondary reconstruction. VSP allows for the utilization of the unaffected anatomical side as a mirror to create a template. This improves the surgeon's ability to achieve the ideal projection and restoration of facial buttresses ${ }^{[15]}$. In addition, the authors recommend superimposing the donor bone segment during the planning session to ensure optimization of desired buttress restoration and projection ${ }^{[15]}$.

\section{DENTAL IMPLANTATION}

Dental restoration is an important component of a patient's quality of life. It has been reported that VSP improves rates of dental rehabilitation ${ }^{[3,24,25]}$. However, at this time, there is not sufficient data to assess longterm outcomes for midface reconstruction or to compare immediate and secondary implantations. The benefits of immediate osseointegrated implantation have been cited as reduced time to dental rehabilitation, with a known elevation of the upfront cost that is offset by the reduction in downstream cost associated with secondary implantation ${ }^{[25]}$. Further research into dental restoration for patients undergoing complex midface reconstruction is warranted. 

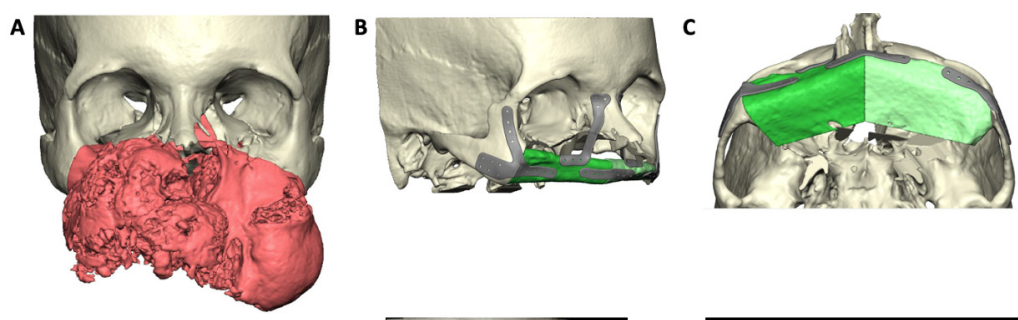

D

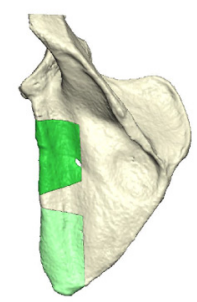

E

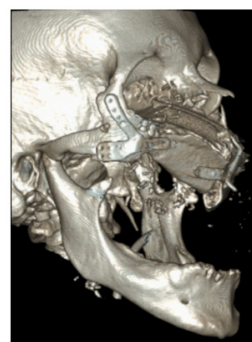

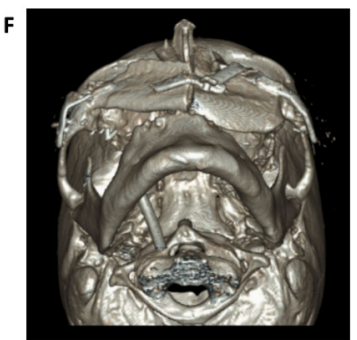

Figure 1. Total midface reconstruction following resection of a large neoplasm. VSP software rendering of neoplasm (red) and the normal facial skeleton (tan) (A). VSP rendering of planned midface reconstruction with scapula bone (red) and custom plates (B, C). VSP rending of scapula bone with planned osteotomy sites (D). Postoperative 3-dimensional rendering of reconstruction (E, F). VSP: Virtual surgical planning.

A

Anatomical Model, Graft Site, Clear

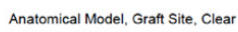

Patient Specific Guide, Bone Graft

Patient Specific Guide, Midface
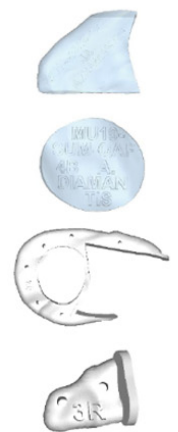

B

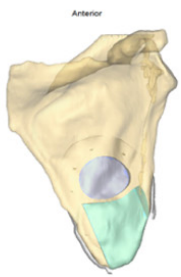

c
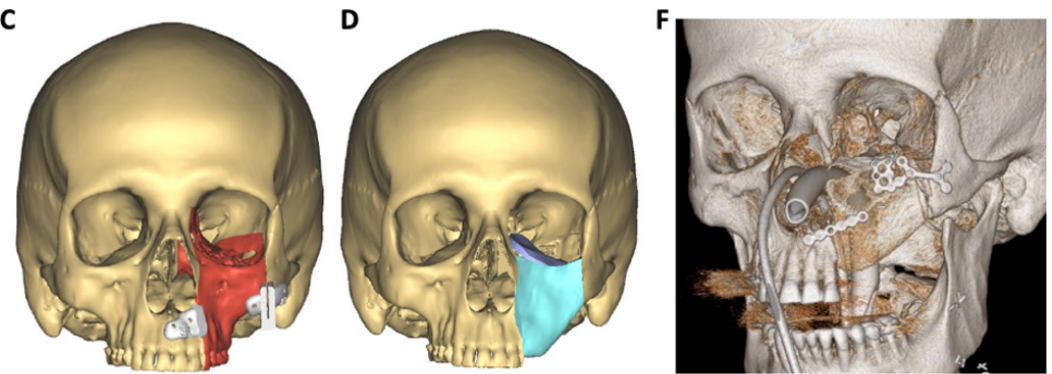

E
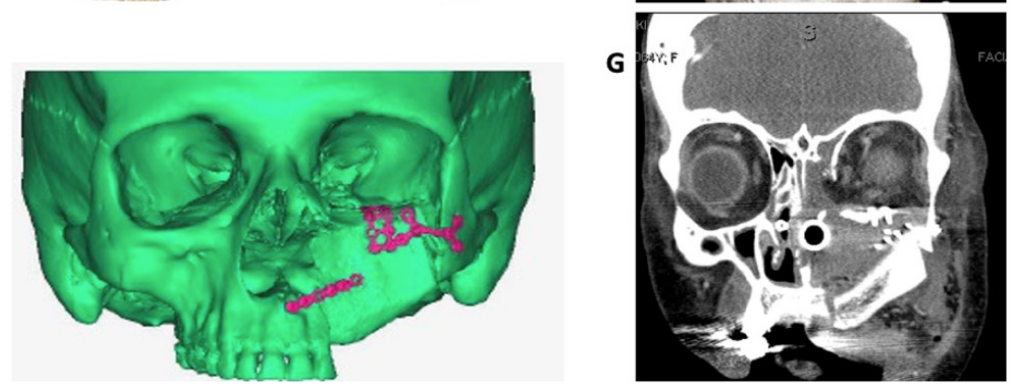

Figure 2. Left midface reconstruction following resection of a large neoplasm. Example of VSP donor site and midface cutting guides and models (A). Anterior and posterior view of the left scapula bone (B). VSP software rendering of neoplasm (red) and the normal facial skeleton (tan) with patient-specific cutting guides in place (C). VSP software rendering of the facial skeleton with planned scapula bone reconstruction of the left anterior maxilla, orbital rim and zygoma (teal), and left orbital floor (purple) (D). Postoperative analysis of reconstructive accuracy (E). 3-Dimensional rendering of postoperative imaging (F). Postoperative coronal CT scan view of the orbital floor and midface reconstruction (G). VSP: Virtual surgical planning.

\section{POSTOPERATIVE}

For assessment of postoperative goals, software programs are available to compare preoperative and postoperative imaging. These programs allow for simultaneous viewing of the imaging with overlay graphics. The distance and desired projection of the donor tissue compared to the native can then be measured $^{[18]}$. Postoperative imaging for assessing osteointegration of the recipient and donor bone interface is recommended at six-to-twelve months following reconstruction. 

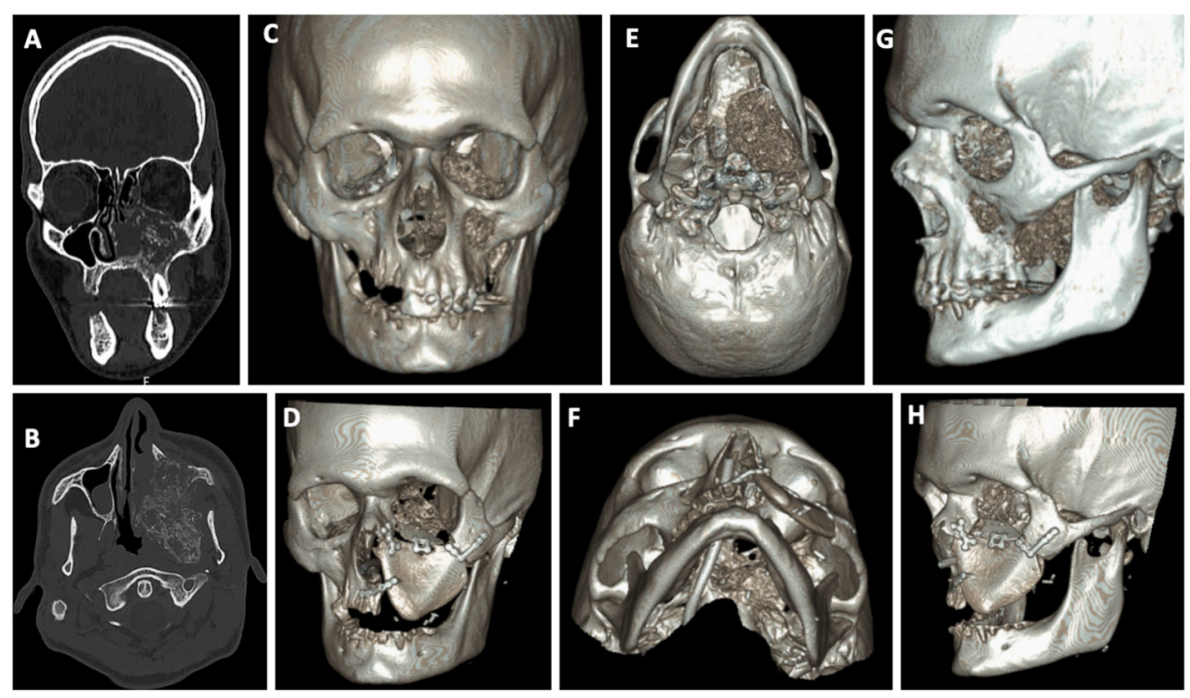

Figure 3. Example of a left midface reconstruction without the aid of virtual surgical planning. Preoperative CT scan, coronal and axial cuts, of midface neoplasm (A, B). 3-Dimensional (3D) rendering of the anterior facial skeleton and midface neoplasm (C) and reconstruction with scapula bone (D). Inferior view of the $3 D$ rendering of the midface neoplasm (E) and scapula reconstruction (F). Lateral view of the 3D rendering of the midface neoplasm $(\mathrm{G})$ and scapula reconstruction $(H)$.

\section{COMPARISONS: VSP VS. NON-VSP}

There are limited publications directly comparing outcomes between VSP and non-VSP midface reconstructions. No differences were found in the operative durations between the two cohorts ${ }^{[1,26]}$. Utilization of VSP resulted in a higher percentage of bone contact between donor and recipient bone compared to the non-VSP cohort ${ }^{[18]}$. On postoperative analysis, VSP improved restoration of the midface subunits and resulted in a more accurate approximation with normal bone anatomy ${ }^{[18]}$.

\section{LIMITATIONS AND CRITIQUES}

Current limitations and critiques of VSP include the need for multiple preoperative steps and manipulations, the high cost of outsourcing, and extra healthcare expense. Planning sessions are often timeintensive and may be disruptive and unfeasible for surgeons. Procedure-specific costs (hardware, equipment, etcetera) have been estimated at \$8000-10,000 for an outsourced VSP compared to \$3000-3700 for the traditional approaches ${ }^{[25-27]}$. The VSP related cost may be justified if it was offset by reduced operative times, complications, length of stay, and 30-day readmission rates ${ }^{[24,28]}$. Further research is warranted in this area to determine the true cost-benefit of VSP for midface reconstruction.

Given the numerous benefits of this technology, it would be advantageous for outsourcing companies to make VSP more readily available and at a lower cost to surgeons. These provisions might allow for its more seamless integration into clinical practice. Some surgeons prefer to utilize internal software and 3D printers for planning and guides, which allows for less expensive options with a reduction in manufacturing time $^{[29-33]}$. While more cost-effective, this method is more demanding of the surgeon's time and requires surgeon experience and comfort with the software. The internal systems typically allow for a 3D model to be printed. The surgeon will then pre-bend plates to the model, versus the custom-made plates that are available from third-party companies. The custom-made plates are typically stronger and designed to maximize bony interfaces and screw placements. 


\section{DISCUSSION}

VSP is a compliment to the reconstructive plan and is best viewed as an additional tool for the reconstructive surgeon to maximize functional outcome and limit operative time. The $3 \mathrm{D}$ modeling can be utilized as an adjunct in complex maxillary reconstruction, improving the precision and accuracy of free flap reconstruction $s^{[1,6,7,9,18]}$. For the midface, an area that is crucial both functionally and aesthetically, millimeters can make profound differences in form and function. With VSP, bone reconstruction is planned to maximize bone-to-bone interface and improve osseointegration. A mirror image of the unaffected side can be used when there is significant distortion or destruction of the anatomy on the operative side to model and act as a template. VSP is also valuable in calculating soft tissue volume loss to aid in flap design and donor site selection. The 3D models can be available intraoperatively and are of particular use if ablative margins differ from the original plan.

The degree of midface reconstruction, and subsequently the complexity, is dependent on the extent of the defect. This varies from patient to patient and requires a solid fundamental understanding of the 3D space that encompasses the midface. VSP may be a valuable aid to preoperatively assess anticipated defects and subsequently evaluate the reconstructive needs. Common goals of surgical reconstruction of the midface include support for the orbit and/or anterior skull base, prevention of oroantral or oronasal fistulas, mirrored symmetry for an optimal cosmetic outcome, and soft tissue contouring, maintenance of oral mastication, preservation of vision, and adequate soft tissue suspension. VSP improves the precision of osteotomies and the efficacy of the reconstruction ${ }^{[18]}$. Proponents of the utilization of VSP cite shorter operative times, with the greatest reduction in operative times if VSP included the design and use of cutting guides $^{[7,34]}$.

The soft tissues overlying the midfacial skeleton are thin, making concealing bony asymmetry in the reconstructed midface difficult. In addition, the thickness of the bone in this region makes robust osseointegration challenging. Furthermore, bacteria that are naturally present within the oral and nasal cavities are in close approximation with the reconstructive hardware, increasing the risk for postoperative infection, wound breakdown, or hardware exposure.

Previous publications of midface reconstruction have found that utilization of VSP improved bone contact between donor segments and the recipient's bone, and resulted in improved buttress restoration compared to reconstructions that did not utilize $\mathrm{VSP}^{[\gamma, 18]}$. On assessment of their postoperative imaging, the utilization of VSP was associated with a reduction in the degree of deviation in greater than $80 \%$ of measurements. This resulted in a higher percentage of bone contact between donor and recipient bone in patients reconstructed with free flaps in the VSP cohort compared to the non-VSP cohort ${ }^{[18]}$. The authors concluded that the use of VSP improved restoration of the subunits for midface reconstruction, allowed for a more accurate approximation to the normal bone anatomy, and improved cosmetic outcomes ${ }^{[18]}$. This technology is particularly useful when the normal anatomy has been distorted by malignancy, prior surgery, trauma, or is congenitally absent.

Free tissue transfers provide vascularized soft tissue and bone that is advantageous for reconstructing complex midface defects. With an improved understanding of free tissue transfers, they have been used with increasing frequency for midface reconstructions in the past two decades ${ }^{[11]}$. VSP is considered an adjunct to midface reconstruction that has further improved our understanding of recipient and donor site anatomy and their 3D interface. The advancement of VSP has allowed for further optimization as surgeons join with engineers to utilize preoperative imaging with computer-aided design and manufacturing. 


\section{DECLARATIONS}

\section{Acknowledgments}

Not applicable.

\section{Authors' contributions}

Made substantial contributions to conception and design of the study, analysis and interpretation, composition of manuscript, and final approval of manuscript: Sweeny L, Fuson A, Curry JM

\section{Availability of data and materials}

Not applicable.

\section{Financial support and sponsorship}

None.

\section{Conflicts of interest}

All authors declared that there are no conflicts of interest.

\section{Ethical approval and consent to participate}

Not applicable.

\section{Consent for publication}

Not applicable.

\section{Copyright}

(c) The Author(s) 2021.

\section{REFERENCES}

1. Bender-Heine A, Wax MK. Reconstruction of the Midface and Palate. Semin Plast Surg 2020;34:77-85. DOI PubMed PMC

2. Breik O, Idle M, Martin T, Praveen P, Parmar S. Three-dimensional computer-assisted surgical planning and manufacturing in complex maxillary reconstruction. Atlas Oral Maxillofac Surg Clin North Am 2020;28:151-64. DOI PubMed

3. Kääriäinen M, Kuuskeri M, Gremoutis G, Kuokkanen H, Miettinen A, Laranne J. Utilization of three-dimensional computer-aided preoperative virtual planning and manufacturing in maxillary and mandibular reconstruction with a microvascular fibula flap. $J$ Reconstr Microsurg 2016;32:137-41. DOI PubMed

4. Ritto FG, Schmitt ARM, Pimentel T, Canellas JV, Medeiros PJ. Comparison of the accuracy of maxillary position between conventional model surgery and virtual surgical planning. Int J Oral Maxillofac Surg 2018;47:160-6. DOI PubMed

5. Sharaf B, Levine JP, Hirsch DL, Bastidas JA, Schiff BA, Garfein ES. Importance of computer-aided design and manufacturing technology in the multidisciplinary approach to head and neck reconstruction. J Craniofac Surg 2010;21:1277-80. DOI PubMed

6. Stokbro K, Bell RB, Thygesen T. Patient-specific printed plates improve surgical accuracy in vitro. J Oral Maxillofac Surg 2018;76:2647. :e1-9. DOI PubMed

7. Wang YY, Fan S, Zhang HQ, Lin ZY, Ye JT, Li JS. Virtual surgical planning in precise maxillary reconstruction with vascularized fibular graft after tumor ablation. J Oral Maxillofac Surg 2016;74:1255-64. DOI PubMed

8. Witjes MJH, Schepers RH, Kraeima J. Impact of 3D virtual planning on reconstruction of mandibular and maxillary surgical defects in head and neck oncology. Curr Opin Otolaryngol Head Neck Surg 2018;26:108-14. DOI PubMed

9. Largo RD, Garvey PB. Updates in head and neck reconstruction. Plast Reconstr Surg 2018;141:271e-85e. DOI PubMed

10. Hanasono MM, Jacob RF, Bidaut L, Robb GL, Skoracki RJ. Midfacial reconstruction using virtual planning, rapid prototype modeling, and stereotactic navigation. Plast Reconstr Surg 2010;126:2002-6. DOI PubMed

11. Alam D, Ali Y, Klem C, Coventry D. The evolution of complex microsurgical midface reconstruction: a classification scheme and reconstructive algorithm. Facial Plast Surg Clin North Am 2016;24:593-603. DOI PubMed

12. Wilkman T, Husso A, Lassus P. Clinical comparison of scapular, fibular, and iliac crest osseal free flaps in maxillofacial reconstructions. Scand J Surg 2019;108:76-82. DOI PubMed

13. Connolly TM, Sweeny L, Greene B, Morlandt A, Carroll WR, Rosenthal EL. Reconstruction of midface defects with the osteocutaneous radial forearm flap: Evaluation of long term outcomes including patient reported quality of life. Microsurgery 2017;37:752-62. DOI PubMed

14. Garvey PB, Chang EI, Selber JC, et al. A prospective study of preoperative computed tomographic angiographic mapping of free fibula osteocutaneous flaps for head and neck reconstruction. Plast Reconstr Surg 2012;130:541e-9e. DOI PubMed PMC 
15. Shen Y, Sun J, Li J, Li MM, Huang W, Ow A. Special considerations in virtual surgical planning for secondary accurate maxillary reconstruction with vascularised fibula osteomyocutaneous flap. J Plast Reconstr Aesthet Surg 2012;65:893-902. DOI PubMed

16. Swendseid B, Kumar A, Sweeny L, et al. Natural history and consequences of nonunion in mandibular and maxillary free flaps. Otolaryngol Head Neck Surg 2020;163:956-62. DOI PubMed

17. Ferrari S, Ferri A, Bianchi B. Scapular tip free flap in head and neck reconstruction. Curr Opin Otolaryngol Head Neck Surg 2015;23:115-20. DOI PubMed

18. Swendseid BP, Roden DF, Vimawala S, et al. Virtual surgical planning in subscapular system Free flap reconstruction of midface defects. Oral Oncol 2020;101:104508. DOI PubMed

19. Lanzer M, Gander T, Gratz K, Rostetter C, Zweifel D, Bredell M. Scapular free vascularised bone flaps for mandibular reconstruction: are dental implants possible? J Oral Maxillofac Res 2015;6:e4. DOI PubMed PMC

20. Burgess M, Leung M, Chellapah A, Clark JR, Batstone MD. Osseointegrated implants into a variety of composite free flaps: A comparative analysis. Head Neck 2017;39:443-7. DOI PubMed

21. Blumberg JM, Walker P, Johnson S, et al. Mandibular reconstruction with the scapula tip free flap. Head Neck 2019;41:2353-8. DOI PubMed

22. Clark JR, Vesely M, Gilbert R. Scapular angle osteomyogenous flap in postmaxillectomy reconstruction: defect, reconstruction, shoulder function, and harvest technique. Head Neck 2008;30:10-20. DOI PubMed

23. Barrett MG, de Rijk WG, Burgess JO. The accuracy of six impression techniques for osseointegrated implants. J Prosthodont 1993;2:75-82. DOI PubMed

24. Avraham T, Franco P, Brecht LE, et al. Functional outcomes of virtually planned free fibula flap reconstruction of the mandible. Plast Reconstr Surg 2014;134:628e-34e. DOI PubMed

25. Tamaki A, Seim NB, Valentin S, et al. The use of medical modeling in microvascular free tissue transfer reconstruction with osseointegrated implantation in complex midface defects. Oral Oncol 2020;110:104982. DOI PubMed

26. Mazzola F, Smithers F, Cheng K, et al. Time and cost-analysis of virtual surgical planning for head and neck reconstruction: a matched pair analysis. Oral Oncol 2020;100:104491. DOI PubMed

27. Fatima A, Hackman TG, Wood JS. Cost-effectiveness analysis of virtual surgical planning in mandibular reconstruction. Plast Reconstr Surg 2019;143:1185-94. DOI PubMed

28. Chang EI. Long-term operative outcomes of preoperative computed tomography-guided virtual surgical planning for osteocutaneous free flap mandible reconstruction. Plast Reconstr Surg 2016;138:559e-60e. DOI PubMed

29. Spaas C, Lenssen O. Economic analysis of a low-cost virtual surgical planning protocol for mandibular reconstruction: a case series. Br J Oral Maxillofac Surg 2019;57:743-8. DOI PubMed

30. Sharkh H, Makhoul N. In-house surgeon-led virtual surgical planning for maxillofacial reconstruction. J Oral Maxillofac Surg 2020;78:651-60. DOI PubMed

31. Rommel N, Kesting MR, Rohleder NH, Bauer FMJ, Wolff KD, Weitz J. Mandible reconstruction with free fibula flaps: Outcome of a cost-effective individual planning concept compared with virtual surgical planning. J Craniomaxillofac Surg 2017;45:1246-50. DOI PubMed

32. Mendez BM, Chiodo MV, Patel PA. Customized "in-office" three-dimensional printing for virtual surgical planning in craniofacial surgery. J Craniofac Surg 2015;26:1584-6. DOI PubMed

33. Smithers FAE, Cheng K, Jayaram R, Mukherjee P, Clark JR. Maxillofacial reconstruction using in-house virtual surgical planning. ANZ J Surg 2018;88:907-12. DOI PubMed

34. Barr ML, Haveles CS, Rezzadeh KS, et al. Virtual surgical planning for mandibular reconstruction with the fibula free flap: a systematic review and meta-analysis. Ann Plast Surg 2020;84:117-22. DOI PubMed 